\title{
EL GLUFOSINATO DE AMONIO ALTERA LA CALIDAD Y EL ADN DE LOS ESPERMATOZOIDES DE RATÓN
}

\author{
Cecilia GONZÁLEZ CALIXTO ${ }^{1}$, María Elena MORENO GODÍNEZ ${ }^{2}$, Mireya MARURIS REDUCINDO ${ }^{3}$, \\ María Isabel HERNÁNDEZ OCHOA ${ }^{4}$, Ma. Betzabet QUINTANILLA VEGA ${ }^{4}$ y \\ Mayrut Osdely URIOSTEGUI ACOSTA ${ }^{3 *}$
}

${ }^{1}$ Facultad de Enfermería No. 2, Universidad Autónoma de Guerrero. Paseo de la Cañada, Alta Progreso, Acapulco, Gro. C.P. 39610

${ }^{2}$ Facultad de Ciencias Químico Biológicas de la Universidad Autónoma de Guerrero. Av. Lázaro Cárdenas, El Centenario, Chilpancingo de los Bravo, Gro. C.P. 39090

${ }^{3}$ Escuela Superior de Ciencias Naturales de la Universidad Autónoma de Guerrero. Ex rancho Shalako, Carr. Nal. Chilpancingo-Petaquillas, Gro. C.P. 39105

${ }^{4}$ Departamento de Toxicología, Centro de Investigación y de Estudios Avanzados del Instituto Politécnico Nacional (Cinvestav), Instituto Politécnico Nacional 2508, Col. San Pedro Zacatenco, Ciudad de México, México C.P. 07360

*Autor para correspondencia: mayruturiostegui@gmail.com

(Recibido enero 2017; aceptado diciembre 2017)

Palabras clave: organofosforados, calidad espermática, daño genético

\section{RESUMEN}

El glufosinato de amonio (GLA) es un herbicida organofosforado que se utiliza para el control de malezas. La exposición a GLA induce incrementos en la incidencia de partos prematuros, anormalidades y abortos en hembras preñadas, así como un retraso en el desarrollo del cigoto de preimplantación. Sin embargo, se desconoce si el GLA tiene como blanco de toxicidad a las células espermáticas durante la última fase de su desarrollo y de maduración. Este estudio evaluó los efectos del GLA sobre la calidad de los espermatozoides y del ADN espermático después de la exposición durante dos etapas cruciales, la de espermátida y la de maduración epidídimal. Ratones macho adultos de la cepa ICR fueron administrados diariamente con GLA [5, 7.5 y $10 \mathrm{mg} / \mathrm{kg}$ de peso corporal/v.o./5- ó 15-días de tratamiento (dt)], y sacrificados $24 \mathrm{~h}$ post-tratamiento. Los espermatozoides fueron obtenidos de la cola del epidídimo-conducto deferente y después fueron utilizados para evaluar la calidad espermática y el daño al ADN. A los 5- ó 15-dt, las dosis de 5, 7.5 y $10 \mathrm{mg} / \mathrm{kg} / \mathrm{d}$ de GLA, disminuyeron significativamente los parámetros de calidad espermática. Asimismo, a los 5-dt, la dosis de $10 \mathrm{mg} / \mathrm{kg} / \mathrm{d}$ incrementó significativamente el \%DFI (por sus siglas en inglés, "DNA Fragmentation Index"), mientras que a los 15 -dt las dosis de 7.5 y $10 \mathrm{mg} / \mathrm{kg} / \mathrm{d}$ incrementaron significativamente la media de \%DFI y \%HSD (por sus siglas en inglés, "High DNA stainability" o Alta fluorescencia del ADN). Estos datos sugieren que las espermátidas tempranas y los espermatozoides maduros son blancos de la exposición a GLA.

Key words: organophosphorus, quality sperm, genetic damage 


\begin{abstract}
Glufosinate ammonium (GLA) is an organophosphorus herbicide used to control weeds. GLA exposure increases premature parturition, abnormalities, and stillbirths in pregnant females, as well as a delay in preimplantation zygote development. However, it is unkown whether GLA targets sperm cells during last stages of development and maturation, and if it damages sperm quality. This study evaluated the effects of GLA exposure on sperm quality and sperm DNA following exposure during two crucial stages of sperm development, the spermatid and final maturation. Adult male ICR mice were administered daily with $5,7.5$ and $10 \mathrm{mg} / \mathrm{kg} / \mathrm{d}$ GLA for 5 - or 15 -days of treatment (dt), and euthanized $24 \mathrm{~h}$ post-treatment. Spermatozoa were collected from cauda epididymis-vas deference and were then used to evaluate sperm quality and DNA damage. At 5- or 15-dt, the doses of $5,7.5$ and $10 \mathrm{mg} / \mathrm{kg} / \mathrm{d}$ GLA significantly decreased sperm quality parameters. Also, at 5-dt, the dose of $10 \mathrm{mg} / \mathrm{kg} / \mathrm{d}$ GLA significantly increased the \% DFI (DNA fragmentation index), whereas at 15-dt the doses of 7.5 and $10 \mathrm{mg} / \mathrm{kg} / \mathrm{d}$ significantly increased the mean of \% DFI and \%HDS (For its initials in English: High DNA stainability). These data suggest that spermatid and mature sperm cells are targeted by GLA.
\end{abstract}

\section{INTRODUCCIÓN}

Los plaguicidas son agroquímicos ampliamente usados en países en desarrollo para el control de plagas, incluidos los vectores de enfermedades humanas y de animales (USEPA 2010). A pesar de la importancia económica de estos productos, es necesario destacar que su aplicación indiscriminada puede ocasionar daño a la salud de poblaciones expuestas (OMS 1990). El glufosinato de amonio (GLA) es un herbicida foliar, químicamente un organofosforado (OF) no selectivo, que se utiliza para controlar el crecimiento de malezas post-emergentes. La Organización Mundial de la Salud (OMS) lo clasifica como moderadamente peligroso (Grupo II). Está registrado para su uso en cultivos y zonas no cultivables en: plantas ornamentales y una variedad de áreas industriales, residenciales y públicas (INCHEM 1991, NIH 2015). El GLA está disponible en muchos países, incluyendo Estados Unidos, Canadá, Alemania, Reino Unido y Corea. Su uso ha aumentado gradualmente, acompañado de un incremento en la incidencia de casos de envenenamiento en los países asiáticos como Corea, Japón y Taiwán (Hirose et al. 1999, Matsumura et al. 2001, Mao et al. 2012).

En humanos, la intoxicación por GLA a nivel del sistema nervioso central se atribuye principalmente a su estructura análoga con el ácido glutámico y su potencial para inhibir a la descarboxilasa del ácido glutámico, que lleva a una disminución del neurotransmisor ácido gamma-aminobutírico (GABA). Sin embargo, no todos sus mecanismos de toxicidad están bien caracterizados (Fagg y Lanthorn 1985,
Lluís et al. 2008). En ratas, el NOAEL establecido para GLA es de $14 \mathrm{mg} / \mathrm{kg}$ de peso corporal (IPCS 1991). La DL50 establecida en ratones de la cepa ICR es de $436 \mathrm{mg} / \mathrm{kg}$ de peso corporal, mientras que la dosis reportada para intentos suicidas en humanos es aproximadamente $13.9 \mathrm{mg} / \mathrm{kg}$ de peso corporal (IPCS 1991, Schulte-Hermann et al. 2006, Mao et al. 2012).

Se ha demostrado que la exposición ambiental y ocupacional a plaguicidas se asocia con alteraciones en la calidad espermática y daño genético (Martenies y Perry 2013). En agricultores italianos se asoció la exposición a una mezcla de plaguicidas, entre ellos el GLA, con la generación de daño al ADN en células somáticas y con la presencia de aductos 8-hidroxiguanosina (8-oxodG) en células de sangre periférica (Cortes-Genchi et al. 2008, Koureas et al. 2014, Locia-Morales 2014). Otro estudio realizado en trabajadores chinos de una fábrica de plaguicidas (etil-paratión, metil-paratión y metamidofos) reportó una reducción en la concentración y motilidad espermática (Padungtod et al. 2000). Por su parte, Sánchez-Peña et al. (2004) reportaron la presencia de células inmaduras y alteración en la estructura de la cromatina de los espermatozoides de agricultores de La Comarca Lagunera, México expuestos a una mezcla de plaguicidas. Finalmente, Pérez-Herrera et al. (2008) reportaron una disminución en la calidad del semen y daño al ADN en agricultores del estado de Yucatán, afectando los distintos estadios de maduración de las células espermáticas.

Se sabe que otros plaguicidas OF, como el metil-paratión y el metamidofos dañan al ADN de 
espermatozoides maduros y de células espermáticas en desarrollo (Piña-Guzmán et al. 2005, UriósteguiAcosta et al. 2014a). Un espermatozoide que posee ADN dañado puede tener una capacidad fertilizante deficiente, o bien, pueden presentarse problemas para el desarrollo del blastocisto, y en consecuencia para la implantación en el útero (Lopes et al. 1998, Ahmadi y Ng 1999, Tomlinson et al. 2001, Hales et al. 2005). Aunque el GLA es ampliamente utilizado en diversas regiones de México y otros países, sus efectos potenciales sobre la reproducción han sido poco estudiados. Un estudio realizado en ratones hembra reportó que la exposición a 360 ppm de GLA causa pérdidas de los cigotos que están listos para implantarse, incluso, de aquéllos que ya se implantaron en el útero (Ebert et al. 1990, Schulte-Hermann et al. 2006). Otro estudio realizado también en ratones hembra reportó que la exposición a GLA ( $58 \mu \mathrm{L} / \mathrm{kg}$ de peso $)$ impide que los ovocitos fertilizados alcancen la etapa de blastocisto (Fabián et al. 2011). Finalmente, un estudio realizado en conejos hembra reportó que la exposición a $20 \mathrm{mg} /$ $\mathrm{kg}$ de peso/d de GLA aumenta la incidencia de partos prematuros y de abortos (Baeder y Kramer 1984). Sin embargo, hasta la fecha no hay estudios que hayan evaluado si el GLA altera la calidad de los espermatozoides en dos etapas cruciales para la capacidad fertilizante, lo cual podría explicar parcialmente los mecanismos de toxicidad de este plaguicida OF. Por lo tanto, el objetivo de este trabajo fue evaluar el efecto del GLA sobre la calidad de los espermatozoides y de su ADN después de haber sido expuestos mientras se encontraban en la fase de espermátida temprana o en la fase de espermatozoide en proceso de maduración epidídimal.

\section{MATERIALES Y MÉTODOS}

Se utilizaron ratones machos de la cepa ICR-CD1 de 12 semanas de edad del Bioterio del IBT-UNAM. Los animales se mantuvieron en condiciones ideales a una temperatura de $18-23{ }^{\circ} \mathrm{C}$, con humedad relativa del $40-70 \%$, con un régimen de alimentación y agua esterilizada y en ciclos de luz/oscuridad de 12 $h$. Los grupos de ratones $(n=10)$ se asignaron aleatoriamente; los testigo $(\mathrm{n}=4)$ recibieron el vehículo (agua) y los tratados $(\mathrm{n}=6)$ recibieron el GLA $(97 \%$ de pureza; Sigma, Saint Louis, MO, USA) en dosis de exposición diarias de $5,7.5 \mathrm{o} 10 \mathrm{mg} / \mathrm{kg}$ de peso corporal/v.o./5- ó 15-días de tratamiento (dt). Las dosis de exposición fueron seleccionadas con base en lo establecido por Calas et al. (2008), el tiempo de exposición de 5-dt es debido a que, en el ratón, los espermatozoides permanecen en el epidídimo entre 5 y 7 días, mientras que a los 15 -d se da la remoción de histonas y el cambio de proteínas de transición por protaminas (Eddy y O'brien 1989, Boussouar et al. 2014). Los animales fueron sacrificados por dislocación cervical $24 \mathrm{~h}$ después de la última administración. Dos experimentos independientes fueron realizados por cada dosis. Todos los procedimientos con animales fueron aprobados por el Comité de Ética Institucional de la UAGro de conformidad con las Directrices Internacionales para el uso y cuidado de animales de laboratorio. Todos los reactivos adquiridos fueron de Sigma-Aldrich.

\section{Obtención de la muestra espermática}

Los espermatozoides se obtuvieron por perfusión de la cola del epidídimo-conducto deferente, se colocaron en solución salina $0.9 \%$ y se mantuvieron a una temperatura de $37^{\circ} \mathrm{C}$.

\section{Evaluación de calidad espermática}

La calidad espermática se determinó con base en los parámetros establecidos por la OMS (2010), evaluándose la viabilidad, motilidad y cuenta. La morfología espermática se valoró con base a lo establecido por Wyrobek et al. (1983). Para ello se contabilizaron 200 células en cada prueba y los valores individuales por animal se expresaron en porcentaje. Posteriormente, los datos se expresaron como porcentaje respecto al grupo testigo.

\section{Evaluación de la integridad del ADN y estructura de la cromatina espermática por citometría de flujo (SCSA)}

Se empleó la técnica Sperm Chromatin Structure Assay (SCSA), modificada por Evenson (2016). La técnica de SCSA evalúa la susceptibilidad de la cromatina espermática a la desnaturalización ácida in situ. Las muestras fueron incubadas con el fluorocromo naranja de acridina (NA), el cual posee propiedades metacromáticas que le permiten intercalarse entre las bases del ADN de doble cadena y emitir una fluorescencia verde (ADN no desnaturalizado) y cuando se intercala con el ADN de cadena sencilla emite una fluorescencia roja (ADN desnaturalizado). Se evaluaron el Índice de fragmentación del ADN (\% DFI por sus siglas en inglés), y la Alta fluorescencia del ADN (\% HDS por sus siglas en inglés), los cuales representan el daño al ADN y el porcentaje de células con una cromatina inmadura o alterada, respectivamente.

Para ello, una alícuota de $2 \times 10^{6}$ espermatozoides se resuspendió en $200 \mu 1$ del amortiguador TNE (Tris- $\mathrm{HCl} 0.01 \mathrm{M}, \mathrm{NaCl} 0.15 \mathrm{M}$ y EDTA $0.01 \mathrm{M}$, 
pH 7.4), y se sónico (Ultrasonic processor GE-130) a un máximo de potencia de $60 \%$ a $4{ }^{\circ} \mathrm{C} / 3 \mathrm{~min}$ para eliminar las células somáticas contaminantes. Posteriormente, a una alícuota de $100 \mu \mathrm{L}$ de esta suspensión $\left(1 \times 10^{6}\right.$ espermatozoides $)$ se le agregaron $200 \mu \mathrm{L}$ de la solución permeabilizante $(\mathrm{HCl} 0.08$ $\mathrm{N}, \mathrm{NaCl} 0.15$ M y Tritón X-100 $0.1 \%, \mathrm{pH} 1.2$ ) y la muestra se dejó reposar sobre una cama de hielo durante 30 seg para facilitar la entrada del fluorocromo a las células. Inmediatamente después se adicionaron $600 \mu \mathrm{L}$ de la solución de tinción (ácido cítrico $0.1 \mathrm{M}, \mathrm{Na}_{2} \mathrm{HPO}_{4} 7 \mathrm{H}_{2} \mathrm{O} 0.2 \mathrm{M}$, EDTA 0.001 $\mathrm{M}, \mathrm{NaCl} 0.15 \mathrm{M}$ y $600 \mu \mathrm{l}$ de NA) y la muestra se dejó reposar en hielo durante $3 \mathrm{~min}$. Posteriormente, la preparación celular fue depositada en un tubo de polipropileno para su análisis en un citómetro de flujo (FACSort Becton Dickinson, CA). Una muestra de referencia fue evaluada en cada corrida como control de calidad. Los datos fueron analizados en el programa SCSASoft ${ }^{\circledR}$ (SCSA Diagnostic, Inc., Brookings, SD).

\section{Análisis estadístico}

Se presenta la media, percentil 75 , máximos y mínimos o la media y el rango de dos experimentos independientes por cada dosis de exposición en ambos tiempos. Las diferencias entre las medianas de los grupos de tratamiento se analizaron con las pruebas de Kruskal-Wallis y U de Wilcoxon. El análisis estadístico se llevó a cabo con el programa Stata 12 (Stata Corp., Collage Station, TX). El valor de $\mathrm{p}<0.05$ fue considerado como estadísticamente significativo.

\section{RESULTADOS}

Peso corporal y relativo de órganos de importancia

El peso corporal y el relativo de algunos órganos disminuyeron significativamente a partir de la dosis de exposición de $7.5 \mathrm{mg} / \mathrm{kg} / \mathrm{d}$, en ambos tiempos de tratamiento. Específicamente, a los 5-dt disminuyó el peso relativo de los riñones, pulmones y testículos, mientras que a los 15-dt disminuyó el peso relativo de pulmones, vesículas seminales, glándulas coagulantes y testículos (Cuadro I).

La exposición a GLA afecta la calidad espermática

Los parámetros de calidad espermática fueron analizados para evaluar la capacidad del GLA para alterar las células germinales. A los 5-dt disminuyó la motilidad $(23,49$ y $31 \%)$ y la viabilidad $(34,50$ y $54 \%)$, y se observó un incremento en las alteraciones morfológicas $(22,25$ y $29 \%)$ con $5,7.5$ y $10 \mathrm{mg} / \mathrm{kg} / \mathrm{d}$, respectivamente comparado con el grupo testigo. A los 15-dt, a las mismas concentraciones, disminuyeron la motilidad $(19,43$ y $44 \%$, respectivamente) y la viabilidad (32, 40 y 48 $\%$, respectivamente), además fueron observadas alteraciones morfológicas (23, 48 y $50 \%$, respectivamente) comparado con el grupo testigo (Fig 1a-c).

\section{La exposición a GLA altera la integridad del ADN y estructura de la cromatina espermática}

Los resultados mostraron que el \% DFI, el cual es un indicador de daño en la integridad del ADN, no se afectó con 5 y $7.5 \mathrm{mg} / \mathrm{kg} / \mathrm{d}$ en ambos tiempos de tratamiento, mientras que sí se incrementó con $10 \mathrm{mg} / \mathrm{kg} / \mathrm{d}$ en 1.9 y 2.2 veces a los 5 - y 15 -dt, respectivamente

CUADRO I. LA EXPOSICIÓN A GLA AFECTA EL PESO CORPORAL Y RELATIVO A LOS 5- Ó 15-DÍAS DE TRATAMIENTO

\begin{tabular}{|c|c|c|c|c|c|}
\hline Grupo & $\begin{array}{l}\text { Peso corporal } \\
\text { (g) }\end{array}$ & $\begin{array}{l}\text { Riñón } \\
(\%)\end{array}$ & $\begin{array}{l}\text { Pulmones } \\
\quad(\%)\end{array}$ & $\begin{array}{l}\text { Vesículas seminales y } \\
\text { Glándulas coagulantes (\%) }\end{array}$ & $\begin{array}{l}\text { Testículos } \\
(\%)\end{array}$ \\
\hline \multicolumn{6}{|c|}{ GLA (mg/kg/d/5-días) } \\
\hline Testigo & $42.4(34.1-52.8)$ & $0.65(0.6-2.3)$ & $0.28(0.2-0.6)$ & $0.38(0.2-0.6)$ & $0.25(0.2-0.4)$ \\
\hline 5 & $42.9(34.5-47.7)$ & $0.69(0.5-0.8)$ & $0.34(0.3-0.4)$ & $0.32(0.2-0.4)$ & $0.27(0.2-0.4)$ \\
\hline 7.5 & $* \S 37(33.2-41.7)$ & $0.69(0.5-0.8)$ & $* \S 0.23(0.2-0.4)$ & $0.28(0.2-0.3)$ & $* \S 0.20(0.1-0.3)$ \\
\hline 10 & $* \S 35.9(30.1-42.4)$ & $* \S 0.52(0.4-0.7)$ & $* \S 0.29(0.2-0.3)$ & $0.29(0.2-0.3)$ & $* \S 0.20(0.2-0.3)$ \\
\hline \multicolumn{6}{|c|}{ GLA (mg/kg/d/15-días) } \\
\hline Testigo & $42.05(37.5-50.2)$ & $0.63(0.5-0.9)$ & $0.3(0.2-0.3)$ & $0.41(0.3-0.7)$ & $0.26(0.2-0.3)$ \\
\hline 5 & $41.75(35.1-45.6)$ & $0.59(0.5-0.8)$ & $0.29(0.3-0.4)$ & $0.41(0.3-0.5)$ & $0.26(0.2-0.3)$ \\
\hline 7.5 & *§39.3 (32.3- 39.3) & $0.56(0.3-0.7)$ & $* 0.25(0.2-0.3)$ & $* 0.28(0.2-0.5)$ & $* \S 0.19(0.1-0.2)$ \\
\hline 10 & *\$37.66 (27.9- 39.2) & $0.68(0.4-0.8)$ & $* 0.28(0.2-0.3)$ & $* 0.26(0.3-0.4)$ & $* \S 0.17(0.1-0.3)$ \\
\hline
\end{tabular}

Los datos representan la mediana y el rango (animales testigo $(n=8)$ y tratados $(n=12)$ por dosis).

$* \mathrm{P}<0.05$ fue considerada significativa comparada contra el testigo de acuerdo a la prueba de Wilcoxon.

$\S$ Diferencia significativa $(\mathrm{p}<0.05)$ comparado con la dosis de $5 \mathrm{mg} / \mathrm{kg} / \mathrm{d}$ de acuerdo a la prueba Kruskal Wallis. 

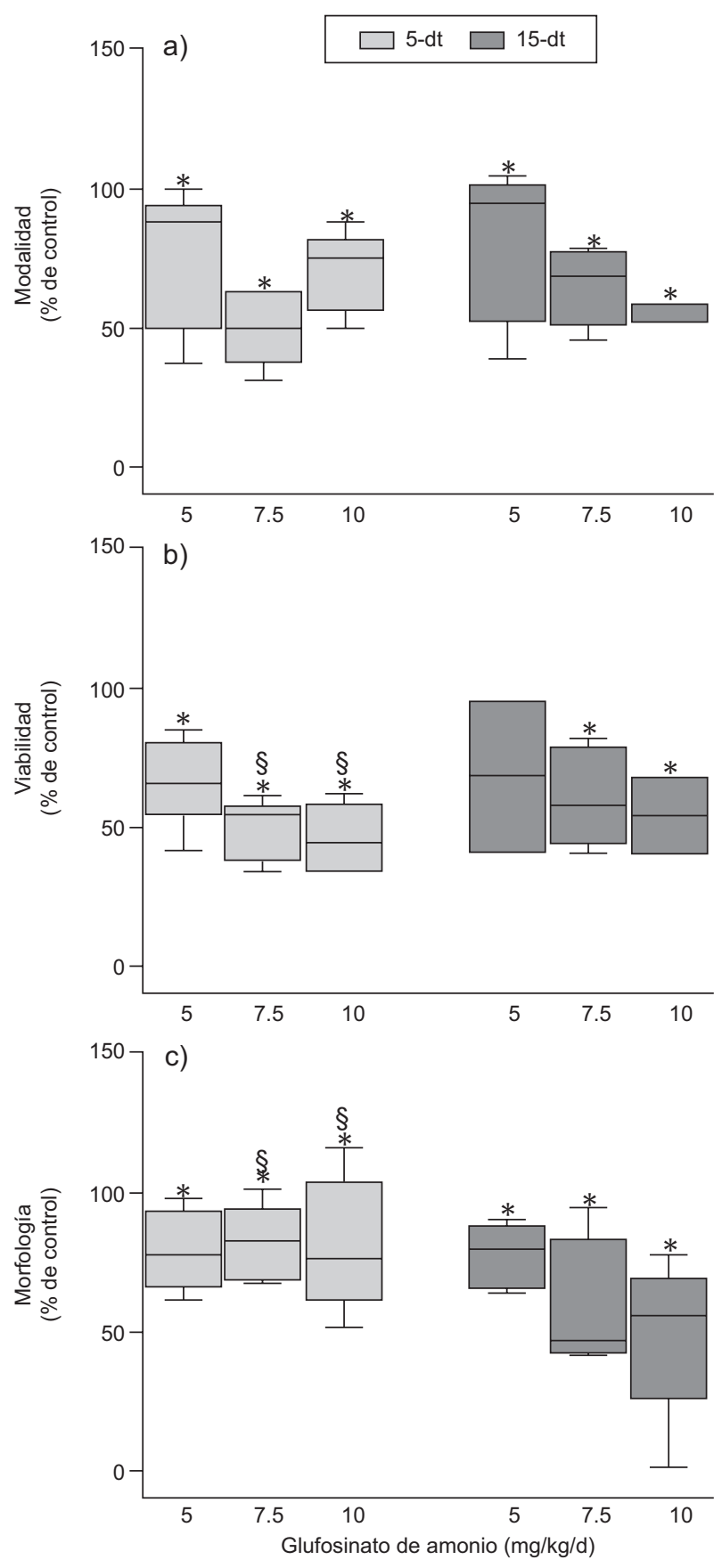

Fig. 1. Parámetros de calidad espermática después de la exposición a GLA. Los espermatozoides fueron colectados 24 $\mathrm{h}$ después de la última administración (5- ó 15-dt) y se evaluó la motilidad (a), viabilidad (b) y morfología (c). Los datos representan la mediana, percentil 75, máximo y mínimo. Los grupos testigo y tratado se conformaron de 8 y 12 ratones, respectivamente, por dosis. *Diferencia significativa comparada al grupo testigo $(\mathrm{p}<0.05)$ de acuerdo a la prueba de Wilcoxon y ${ }^{\S}$ Diferencia significativa $(\mathrm{p}<0.05)$ comparada con el grupo de $5 \mathrm{mg} / \mathrm{kg} / \mathrm{d}$ de acuerdo a la prueba de Kruskal Wallis
(Fig 2a). La condensación de la cromatina (\% HDS) no se modificó con ninguna dosis de exposición a los 5 -dt, pero sí incrementó con 7.5 y $10 \mathrm{mg} / \mathrm{kg} / \mathrm{d}$ (3 y 4 veces, respectivamente) a los 15-dt (Fig $\mathbf{2 b}$ ).

a)

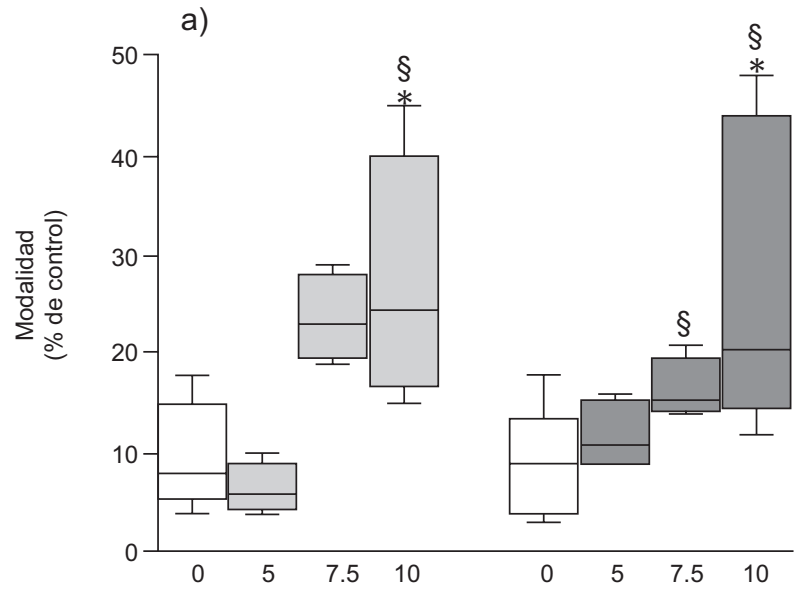

b)

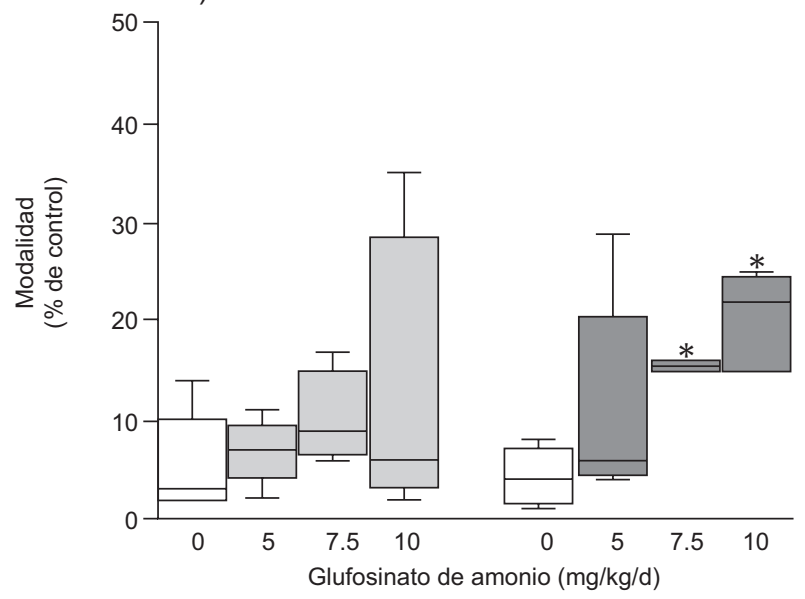

Fig. 2. Efectos sobre la integridad del ADN y cromatina espermática por la exposición a GLA. Los espermatozoides fueron colectados $24 \mathrm{~h}$ después de la última administración (5- ó 15-dt) y se analizaron para evaluar la \%DFI (a) y \%HDS (b), por medio de la técnica de SCSA. Los datos representan la mediana, percentil 75 , máximo y mínimo. Los grupos testigo y tratado se conformaron de 8 y 12 ratones, respectivamente, por dosis. *Diferencia significativa comparada con el grupo testigo $(p<0.05)$ de acuerdo a la prueba de Wilcoxon y ${ }^{\S}$ Diferencia significativa $(\mathrm{p}<0.05)$ comparada con el grupo de $5 \mathrm{mg} / \mathrm{kg} / \mathrm{d}$ de acuerdo a la prueba de Kruskal Wallis.

\section{DISCUSIÓN}

Numerosas investigaciones epidemiológicas realizadas en distintas poblaciones agrícolas del mundo 
han estudiado los efectos asociados con la exposición a plaguicidas (Cortés-Genchi et al. 2007). Estos compuestos son ampliamente utilizados en México y actualmente el GLA es uno de los herbicidas más empleados en algunas áreas del país, representando un problema de salud pública (Pérez-Herrera et al. 2008, Rojas-García et al. 2011, Urióstegui-Acosta et al. 2014b). El GLA ha sido estudiado por sus efectos embriogénicos, dismorfogénicos y teratogénicos, así como por su capacidad de inducir partos prematuros y abortos en hembras expuestas experimentalmente (IPCS, 1999, Schulte-Hermann et al. 2006).

Sin embargo, no se han evaluado los posibles efectos tóxicos del GLA sobre la calidad de los espermatozoides. Por lo tanto, en este estudio se exploraron los efectos del GLA sobre la calidad y alteraciones en la estructura de la cromatina espermática, particularmente la sensibilidad de dos etapas de la espermatogénesis: la espermátida temprana y el espermatozoide en la etapa de maduración epidídimal.

Los resultados de este estudio muestran que la exposición in vivo a GLA induce un daño en la integridad del ADN y la cromatina (\%DFI y \%HDS), así como un deterioro en la calidad espermática en ambos tiempos evaluados, siendo la espermátida temprana (15-dt) la etapa más susceptible al daño. Algunos procesos fisiológicos durante las primeras etapas de la espermatogénesis podrían ser sensibles al daño causado por agentes tóxicos. Uno sería cuando el ADN de la espermátida lleva a cabo un recambio de las histonas somáticas por protaminas (Carrell et al. 2007), mientras que otro proceso sería durante las modificaciones post-traduccionales que pueden favorecer el ataque de agentes exógenos o endógenos sobre el ADN, dando como resultado una alteración en la condensación de la cromatina (Meistrich et al. 2003). Existen reportes en animales de experimentación donde otros plaguicidas OF con acción insecticida, como el diazinón y el quinalfos alteran la calidad espermática y la integridad del ADN cuando los espermatozoides son expuestos durante el estadio de espermátida temprana (Ray et al. 1992, Piña-Guzmán et al. 2005). En el caso del herbicida glifosato, se ha demostrado que, no solo altera los parámetros de calidad y ADN espermáticos, sino que se asocia también con la generación de estrés oxidante (Lopes et al. 2014, Dai et al. 2016). Existen diversas técnicas para evaluar la integridad de la cromatina y el ADN espermático, como la técnica de cromomicina $\mathrm{A}_{3}\left(\mathrm{CMA}_{3}\right)$, el ensayo cometa y SCSA. En el presente estudio, se utilizó la técnica de SCSA por considerarse una herramienta de apoyo elemental en el diagnóstico del potencial fértil masculino (Evenson y Wixon 2005, Piña-Guzmán et al. 2005, Pérez-Herrera et al. 2008, Urióstegui-Acosta et al. 2014a,b). Los datos de SCSA muestran que el parámetro \%DFI se incrementó con la dosis de exposición más alta $(10 \mathrm{mg} / \mathrm{kg} / \mathrm{d})$ en ambos tiempos de tratamiento, mientras que la condensación de la cromatina (\%HDS) se alteró solo a los 15 -dt y a las dosis de 7.5 y $10 \mathrm{mg} / \mathrm{kg} / \mathrm{d}$, comparado con el grupo testigo. Esto sugiere que el GLA tiene como blanco al ADN y a las proteínas nucleares. Este daño puede explicarse por tres mecanismos de toxicidad que se han atribuido a los OF: fosforilación de proteínas, oxidación de proteínas o ADN y alquilación del ADN (es decir, la formación de aductos alquilados). En este sentido, Piña-Guzmán et al. (2005) observaron un incremento en la fosforilación de las protaminas en los residuos de serina de espermátidas y espermatozoides maduros después de la exposición a diazinón. Por otro lado, Urióstegui-Acosta et al. (2014a) reportaron que la exposición a metamidofos incrementó la fosforilación en residuos de serina y tirosina en las células espermáticas en diferentes etapas de la espermatogénesis.

El mecanismo de toxicidad de los OF vía estrés oxidante involucra la oxidación de proteínas de la membrana plasmática, la cual se ha correlacionado con la exposición a OF con la formación de aductos oxidados de 8-hidroxi-2'-deoxiguanosina (8-OHdG) en espermatozoides de ratón, el cual es un marcador de estrés oxidante del ADN (Piña-Guzmán et al. 2006, Monroy-Pérez et al. 2012). Además, en células somáticas se ha encontrado un aumento en la fragmentación del ADN y presencia de 8-OHdG en células mononucleadas procedentes de individuos que residen en poblaciones agrícolas donde se utilizan mezclas de plaguicidas, entre ellos el GLA (Koureas et al. 2014, Locia-Morales et al. 2014). En otras especies como peces (Danio rerio) se observaron alteraciones sobre la integridad del ADN (Lopes et al. 2014). Por otro lado, el mecanismo de alquilación de ácidos nucleicos ha sido ampliamente estudiado en células somáticas donde se ha reportado la formación de aductos de $\mathrm{N}^{7}$ y $O^{6}$ metilguanina, principalmente por la exposición a quinalfos y metamidofos (Eto 1974, Zayed y Mahdi 1987).

Se ha mostrado que los parámetros de calidad espermática se alteran por la exposición a plaguicidas $\mathrm{OF}$ en animales de experimentación. Urióstegui-Acosta et al. (2014a) reportaron un incremento en los parámetros de motilidad, viabilidad y morfología anormal a dosis repetidas de metamidofos en varios estadios de la espermatogénesis (espermatogonia, espermatocito y espermatozoide maduro). De igual manera, la exposición 
a dosis repetidas de fenitrotión alteró la concentración, viabilidad, motilidad y morfología de los espermatozoides (Taib et al. 2014). Por su parte, Piña-Guzmán et al. (2005 y 2006) demostraron que dosis únicas de diazinón y metil-paratión disminuyen la viabilidad, la motilidad y alteran la morfología espermática, además de encontrar una correlación positiva entre las alteraciones en la cromatina y en el ADN espermático. En poblaciones ocupacionalmente expuestas a mezclas de OF se ha demostrado una relación parecida entre las alteraciones sobre la compactación de la cromatina y en el ADN espermático. Pérez-Herrera et al. (2008) reportaron alteraciones en el volumen de eyaculado, cuenta, motilidad y viabilidad espermática en agricultores de Yucatán, y observaron una correlación entre la exposición a plaguicidas con las rupturas del ADN de los espermatozoides. Por su parte, Sánchez-Peña et al. (2004) reportaron alteraciones en la motilidad y viabilidad espermática en agricultores de la Comarca Lagunera. Miranda-Contreras et al. (2013) encontraron una correlación entre las alteraciones en los parámetros de calidad espermática (cuenta, viabilidad y morfología) con el \%DFI. Asimismo, Lopes et al. (2014) observaron alteraciones sobre los parámetros de calidad espermática (motilidad) y el daño al ADN en peces.

Finalmente, la exposición a GLA afectó el peso relativo y de los órganos de importancia como riñones, pulmones, vesículas seminales, glándulas coagulantes y testículos, los cuales son similares a los reportados por Ebert et al. (1994) y pueden atribuirse a la toxicidad general del GLA.

\section{CONCLUSIÓN}

Este estudio demuestra que la exposición subaguda a GLA en ratones causa alteraciones sobre los parámetros de calidad, ADN y cromatina espermática, afectando dos etapas de la espermatogénesis (espermátida temprana y espermatozoide maduro), lo cual podría impactar negativamente en la capacidad fértil masculina. Se requieren estudios adicionales que evalúen si los efectos del GLA observados en este estudio son causados por mecanismos de toxicidad que involucran el estrés oxidante.

\section{AGRADECIMIENTOS}

Los autores agradecen el apoyo de la Red Temática de Toxicología de Plaguicidas (CONACyT-280045) y al Proyecto de Ciencia Básica del
CONACyT (Donativo No. 256595) otorgado a MUA. Los autores agradecen al Dr. Miguel Ángel Gruintal Santos del UACAA-UAGro, por las sugerencias en este manuscrito. A Edith Guinto Ruiz y José de Jesús Sánchez Carlos por su colaboración en las actividades de laboratorio.

\section{REFERENCIAS}

Ahmadi A.y Ng S.C. (1999). Developmental capacity of damaged spermatozoa. Hum. Reprod. 14, 2279-2285. DOI: 10.1093/humrep/14.9.2279

Baeder C. y Kramer M. (1984). Testing for embryotoxicity in Himalayan rabbits following oral administration. Hoechst Report (84.0177).

Boussouar F., Goudarzi A., Buchou T., Shiot H., Barra S., Debernard A. y Arnoult C. (2014). A specific CBP/ p300-dependent gene expression programme drives the metabolic remodelling in late stages of spermatogenesis. Andrology. 2, 351-359.

DOI: $10.1111 / \mathrm{j} .2047$ 2927.2014.00184.

Calas A.G., Richard O., Même S., Beloeil J.C., Doan B.T., Gefflaut T. y Montécot C. (2008). Chronic exposure to glufosinate-ammonium induces spatial memory impairments, hippocampal MRI modifications and glutamine synthetase activation in mice. Neurotoxicology. 29, 740-747. DOI: 10.1016/j.neuro.2008.04.020.

Carrell D.T., Emery B.R. y Hammoud S. (2007). Altered protamine expression and dimished spermatogenesis: what is the link?. Hum. Reprod. Update 13, 313-327. DOI: 10.1093/humupd/dml057.

Cortés-Genchi P., Villegas-Arrizón A., Aguilar-Madrid G., Del Pilar P.R.M., Maruris-Reducindo M. y JuárezPérez C. (2007). Symptom prevalence and pesticide management on agricultural workers. Rev. Med. Inst. Mex. Seguro Soc. 46, 145-152. PMID: 19133185

Dai P., Hu P., Tang J., Li Y. y Li C. (2016). Effect of glyphosate on reproductive organs in male rat. Acta Histochem. 118, 519-526.

DOI: $10.1016 /$ j.acthis.2016.05.009

Degraeve N., Chollet M.C. y Moutschen J. (1985). Mutagenic efficiency of organophosphorus insecticides used in combined treatments. Environ. Health Perspec. 60, 395-398. PMCID: PMC1568550

Ebert E., Leist K.H. y Mayer D. (1990). Summary of safety evaluation toxicity studies of glufosinate ammonium. Food Chem. Toxicol. 28, 339-349. DOI: 10.1016/0278-6915(90)90108-Y

Eddy E. y O'Brien D.A. (1997). 5 Gene Expression during Mammalian Meiosis. Curr. Top. Dev. Biol. 37, 141200. DOI:10.1016/S0070-2153(08)60174-X 
Eto M. (1974). Organosphosphorous pesticides: Orgaic and biological chemistry. CRC press, Cleveland Ohio, 387 pp. DOI: 10.1002/food.19770210224

Evenson D.P. (2016). The Sperm Chromatin Structure Assay (SCSA $\left.{ }^{\circledR}\right)$ and other sperm DNA fragmentation tests for evaluation of sperm nuclear DNA integrity as related to fertility. Anim. Reprod. Sci. 169, 56-75. DOI: 10.1016/j.anireprosci.2016.01.017.

Evenson D.P. y Wixon R. (2005). Environmental toxicants cause sperm DNA fragmentation as detected by the Sperm Chromatin Structure Assay $\left(\mathrm{SCSA}^{\circledR}\right)$. Toxicol. Appl. Pharmacol. 207, 532-537. DOI:10.1016/j.taap.2005.03.021.

Fabian D., Bystriansky J., Burkuš J., Rehák P., Legáth J. y Koppel J. (2011). The effect of herbicide BASTA 15 on the development of mouse preimplantation embryos in vivo and in vitro. Toxicol. In Vitro. 25, 73-79.

DOI: 10.1016/j.tiv.2010.09.009.

Fagg G.E. y Lanthorn T.H. (1985). Cl-/Ca2+-dependent Lglutamate binding sites do not correspond to 2-amino4-phosphonobutanoate-sensitive excitatory amino acid receptors. Br. J. Pharmacol. 86, 743-751.

DOI: 10.1111/j.1476-5381.1985.tb08954.X

Hack R., Ebert E., Ehling G. y Leist K.-H. (1994). Glufosinate ammonium-some aspects of its mode of action in mammals. Food Chem. Toxicol. 32, 461-470.

DOI: 10.1016/0278-6915(94)90043-4

Hales B.F., Aguilar-Mahecha A. y Robaire B. (2005). The stress response in gametes and embryos after paternal chemical exposures. Toxicol. Appl. Pharmacol. 207, 514-520.

DOI: 10.1016/j.taap.2004.12.021.

Hirose Y., Kobayashi M., Koyama K., Kohda Y., Tanaka T., Honda H. y Kikuchi M. (1999). A toxicokinetic analysis in a patient with acute glufosinate poisoning. Hum. Exp. Toxicol. 18, 305-308.

DOI: $10.1191 / 096032799678840110$.

IPCS-INCHEM (1991). Programme on Chemical Safety. 828. Glufosinate ammontium (Pesticide residues in food: 1991 evaluations Part II Toxicology) [en línea] http://www.inchem.org/documents/jmpr/jmpmono/ v91pr12.htm 06-15-15.

Koureas M., Tsezou A., Tsakalof A., Orfanidou T. y Hadjichristodoulou C. (2014). Increased levels of oxidative DNA damage in pesticide sprayers in Thessaly Region (Greece). Implications of pesticide exposure. Sci. Total Environ. 496, 358-364.

DOI: 10.1016/j.scitotenv.2014.07.062.

Lopes F.M., Junior A.S.V., Corcini C.D., da Silva A.C., Guazzelli V.G., Tavares G. y da Rosa C.E. (2014). Effect of glyphosate on the sperm quality of zebrafish Danio rerio. Aquat. Toxicol. 155, 322-326. DOI:10.1016/j.aquatox.2014.07.006.
Lopes S., Jurisicova A., Sun J.G. y Casper R.F. (1998). Reactive oxygen species: potential cause for DNA fragmentation in human spermatozoa. Hum. Reprod. 13, 896-900. DOI: 10.1093/humrep/13.4.896.

Lluís M., Nogué S. y Miró O. (2008). Severe acute poisoning due to a glufosinate containing preparation without mitochondrial involvement. Hum. Exp. Toxicol. 27, 519-524. DOI: 10.1177/0960327108092291.

Locia-Morales D. (2014). Polimorfismo en el gen PON1 y daño del AND en población expuesta ocupacionalmente a plaguicidas organofosforados. Tesis de Maestría. Facultad de Ciencias Quimico BiológicasUAGro. Chilpancingo Guerrero, México, 30 pp.

Mao Y.C., Hung D.Z., Wu M.L., Tsai W.J., Wang L.M., Ger J. y Yang C.C. (2012). Acute human glufosinatecontaining herbicide poisoning. Clin. Toxicol. 50, 396-402. DOI: 10.3109/15563650.2012.676646.

Martenies S.E. y Perry M.J. (2013). Environmental and occupational pesticide exposure and human sperm parameters: a systematic review. Toxicology. 307, 66-73.

DOI: 10.1016/j.tox.2013.02.005

Matsumura N., Takeuchi C., Hishikawa K., Fujii T. y Nakaki T. (2001). Glufosinate ammonium induces convulsion through N-methyl-D-aspartate receptors in mice. Neurosci. Lett. 304, 123-125.

DOI: 10.1016/S0304-3940(01)01765-7.

Meeker J.D., Ryan L., Barr D.B., Herrick R.F., Bennett D.H., Bravo R. y Hauser R. (2004). The relationship of urinary metabolites of carbaryl/naphthalene and chlorpyrifos with human semen quality. Environ. Health Perspect. 112, 1665-1670.

DOI:10.1289/ehp.7234.

Meistrich M.L., Mohapatra B., Shirley C.R. y Zhao M., (2003). Roles of transition nuclear proteins in spermiogenesis. Chromosoma. 111, 483-488.

DOI: $10.1007 / \mathrm{s} 00412-002-0227-z$.

Miranda-Contreras L., Gómez-Pérez R., Rojas G., Cruz I., Berrueta L., Salmen S. y Zavala L. (2013). Occupational exposure to organophosphate and carbamate pesticides affects sperm chromatin integrity and reproductive hormone levels among Venezuelan farm workers. J. Occup. Health. 55, 195-203.

DOI: 10.1539/joh.12-0144-FS.

Monroy-Perez V., Alcántara-Hernández J.A., SolísHeredia M.J., Espinosa-Juárez L. y Quintanilla-Vega B. (2012). Oxidative and genetic damege in germinal and mononuclear mouse cells by methyl-parathion exposure in mice. Toxicologist. 510.

NIH.U.S-National Library of Medicine (2015). TOXNET. Toxicology Data Network. Glufosinateammonium [en línea]. http://chem.sis.nlm.nih.gov/ chemidplus/m/77182-82-2 19/12/2016. 
OMS (1990). Plaguicidas. Informe Técnico No.12. Organización Mundial de la Salud. Ginebra.

OMS (2010). Manual de Laboratorio de la Organización Mundial de la Salud para el examen del semen humano y de la interacción entre el semen y el moco cervical. Editorial Médica Panamericana 6a Edición. Nueva York, 17-22 pp.

Padungtod C., Savitz D.A., Overstreet J.W., Christiani D.C., Ryan L.M. y Xu X. (2000). Occupational pesticide exposure and semen quality among Chinese workers. J. Occup. Environ. Med. 42, 982-992. PMID: 11039162

Pérez-Herrera N., Polanco-Minaya H., Salazar-Arredondo E., Solís-Heredia M., Hernández-Ochoa I., RojasGarcía E. y Quintanilla-Vega B. (2008). PON1Q192R genetic polymorphism modifies organophosphorous pesticide effects on semen quality and DNA integrity in agricultural workers from southern Mexico. Toxicol. Appl. Pharmacol. 230, 261-268.

DOI: 10.1016/j.taap.2008.02.021.

Piña-Guzmán B., Solís-Heredia M. y Quintanilla-Vega B. (2005). Diazinon alters sperm chromatin structure in mice by phosphorylating nuclear protamines. Toxicol. Appl. Pharmacol. 202, 189-198.

DOI: 10.1016/j.taap.2004.06.028.

Piña-Guzmán B., Solís-Heredia M., Rojas-García A., Urióstegui-Acosta M. y Quintanilla-Vega B. (2006). Genetic damage caused by methyl-parathion in mouse spermatozoa is related to oxidative stress. Toxicol. Appl. Pharmacol. 216, 216-224.

DOI: 10.1016/j.taap.2006.05.001.

Ray A., Chatterjee S., Ghosh S., Bhattacharya K., Pakrashi A. y Deb C. (1992). Quinalphos-induced suppression of spermatogenesis, plasma gonadotrophins, testicular testosterone production, and secretion in adult rats. Environ. Res. 57, 181-189.

DOI: 10.1016/S0013-9351(05)80078-7.

Recio R., Robbins W.A., Borja-Aburto V., Moran-Martínez J., Froines J.R., Hernandez R. y Cebrián M.E. (2001). Organophosphorous pesticide exposure increases the frequency of sperm sex null aneuploidy. Environ. Health Perspect. 109, 1237-1240. PMCID: PMC1240505

Rojas-García A.E., Medina-Díaz I.M., de Lourdes Robledo-Marenco M., Barrón-Vivanco B.S., Girón-Pérez, M.I., Velázquez-Fernández, J.B. y Ostrosky-Wegman, P. (2011). Hematological, biochemical effects, and self-reported symptoms in pesticide retailers. J. Occup. Environ. Med. 53, 517-521.

DOI: $10.1097 /$ JOM.0b013e318215fbf2.
Sánchez-Peña L., Reyes B., Lopez-Carrillo L., Recio R., Morán-Martınez J., Cebrian M. y Quintanilla-Vega B. (2004). Organophosphorous pesticide exposure alters sperm chromatin structure in Mexican agricultural workers. Toxicol. Appl. Pharmacol. 196, 108-113. DOI:10.1016/jtaa.2003.11.023.

Schulte-Hermann R., Wogan G.N., Berry C., Brown N.A., Czeizel A., Giavini E. y Neubert D. (2006). Analysis of reproductive toxicity and classification of glufosinateammonium. Regul. Toxicol. Pharmacol. 44, 1-76. DOI:10.1016/j.yrtph.2006.01.008.

Taib I.S., Budin S.B., Ghazali A.R., Jayusman P.A. y Mohamed J. (2014). Fenitrothion alters sperm characteristics in rats: ameliorating effects of palm oil tocotrienol-rich fraction. Exp. Anim. 63, 383-393. DOI: 10.1538/expanim.14-0016.

Tomlinson M., Moffatt O., Manicardi G., Bizzaro D., Afnan M. y Sakkas D. (2001). Interrelationships between seminal parameters and sperm nuclear DNA damage before and after density gradient centrifugation: implications for assisted conception. Hum. Reprod. 16, 2160-2165. DOI: 10.1093/humrep/16.10.2160.

Urióstegui-Acosta M., Hernández-Ochoa I., SánchezGutiérrez M., Piña-Guzmán B., Rafael-Vázquez L., Solís-Heredia M. y Quintanilla-Vega B. (2014a). Methamidophos alters sperm function and DNA at different stages of spermatogenesis in mice. Toxicol. Appl. Pharmacol. 279, 391-400.

DOI:10.1016/j.taap.2014.06.017.

Urióstegui-Acosta M., Hernández-Ochoa I., Solís-Heredia M.J., Martínez-Aguilar G. y Quintanilla-Vega B. (2014b). Comparative effect of technical and commercial formulations of methamidophos on sperm quality and DNA integrity in mice. Environ. Toxicol. 29, 942-949. DOI: 10.1002/tox.21822.

USEPA. (2010). Types of Pesticides. United States Enviromental Protection Agency [en línea] http://www.epa. gov/pesticides/about/types.thm 12/03/2010.

Wyrobek A.J., Gordon L.A., Burkhart J.G., Francis M.W., Kapp R.W., Letz G. y Whorton M.D. (1983). An evaluation of the mouse sperm morphology test and other sperm tests in nonhuman mammals: A report of the US Environmental Protection Agency Gene-Tox Program. Mutat. Res. 115, 1-72. DOI:10.1016/0165-1110(83)90015-5.

Zayed S.M. y Mahdi F.M. (1987). Methylation of guanine in vivo by the organophosphorus insecticide methamidophos. Z. Naturforsch. C. 42, 17-20. DOI:10.1515/znc-1987-1-204. 